\title{
Focal disruption in the wall of the porcelain gallbladder: a sign of gallbladder carcinoma
}

\author{
Mougnyan $\operatorname{Cox}^{1} \cdot$ Manisha Patel $^{1} \cdot$ Upasana Joneja $^{1} \cdot$ Colette Shaw $^{1} \cdot$ \\ Patrick O'Kane ${ }^{1}$
}

Received: 21 November 2016/ Accepted: 28 November 2016/Published online: 8 December 2016 (c) SIMI 2016

A 76-year-old man presented to his primary care physician with a complaint of generalized weakness and unintentional 25-pound weight loss over 3 months. On physical examination, his vital signs were within normal limits, and his examination was notable only for cachexia, mild jaundice, and a firm, tender liver edge palpable four fingerbreadths below the right costal margin. He was admitted to our institution through the emergency department, and CT scan of the abdomen and pelvis (CTAP) was performed (Fig. 1, panel A). On the CTAP, he was found to have a circumferentially calcified gallbladder wall (porcelain gallbladder) with an associated mass extending through the gallbladder fundus and eroding the calcifications in that region. A biopsy of the mass was performed under ultrasound guidance, and the pathology was positive for carcinoma.
Porcelain gallbladder is a term used to describe the appearance of a bluish hue of a brittle calcified gallbladder wall seen at surgery [1]. With the advent of cross-sectional imaging, the term porcelain gallbladder has come to encompass circumferential calcification of the gallbladder wall, usually discovered incidentally on imaging performed for other reasons. Porcelain gallbladder has been shown to be associated with the risk of developing carcinoma of the gallbladder, and prophylactic cholecystectomy has been advocated by multiple authors for this reason [2-4]. More recently, other authors have suggested that association between porcelain gallbladder and gallbladder carcinoma is weaker than originally reported, and cholecystectomy need not be performed for every case [5]. However, in a patient with focal disruption of a calcified gallbladder wall, further imaging is warranted to exclude cancer eroding through the

Mougnyan Cox

mougnyan.cox@gmail.com

1 Thomas Jefferson University, 132 South 10th Street, 1087

Main Building, Philadelphia, PA, USA 


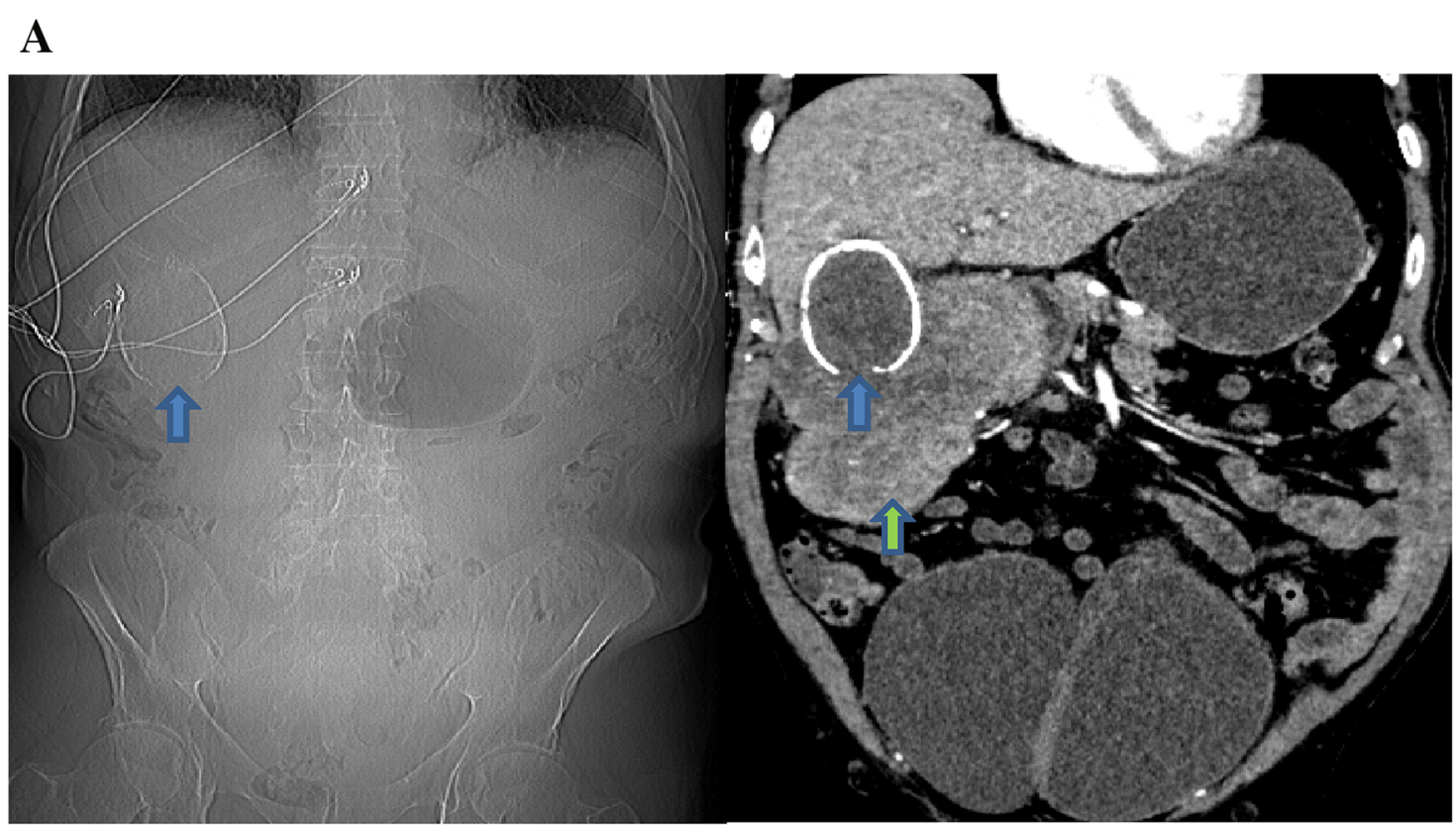

B

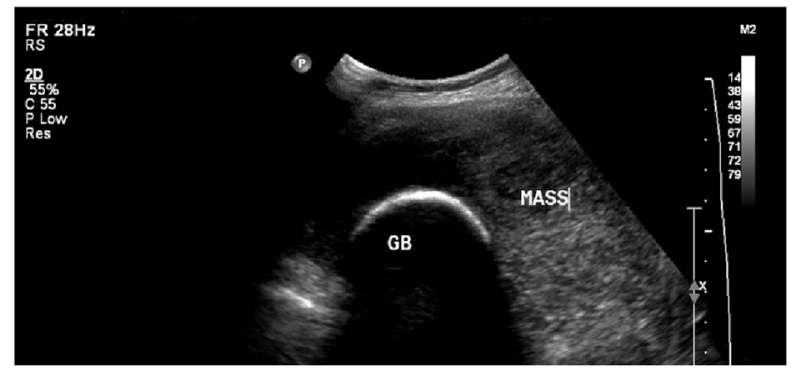

C

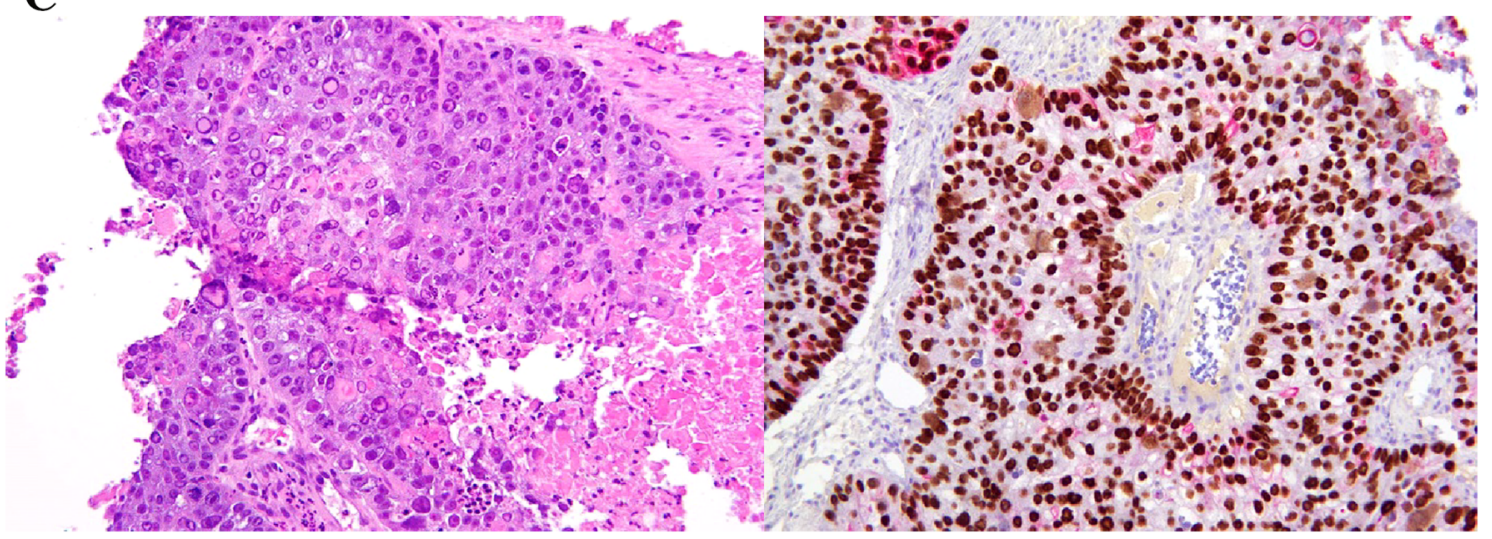


4Fig. 1 Panel A First image is a scout radiograph showing circumferential calcification of the gallbladder, with focal disruption in the region of the gallbladder fundus (blue arrow). Corresponding coronal CTAP with intravenous contrast again shows a calcified gallbladder wall, with focal disruption in the wall caused by a large mass extending through the gall bladder fundus (green arrow). Panel B Right upper quadrant ultrasound performed for biopsy of the mass showed a gallbladder (GB) with a calcified wall, causing posterior acoustic shadowing due to the inability of the ultrasound beam to penetrate the calcifications. A heterogeneous mass was seen next to the gallbladder, and was subsequently targeted for biopsy. Panel C $a$ Gallbladder mass biopsy showing a malignant neoplasm with highly atypical cells with prominent cell membranes and moderate amounts of cytoplasm. The surrounding areas show necrosis, but no identifiable liver parenchyma was present, $\times 200$. $b$ Immunohistochemical staining for p63/CK5/6 shows positive nuclear p63 staining (brown) and patchy positive cytoplasmic CK5/6 staining supporting a squamous differentiation of the carcinoma, $\times 200$. Additional immunohistochemical stains that were positive were CK7 and Hep Par1. Immunohistochemical stains for CDX2/CK20 were negative in this tumor

gallbladder wall and associated calcifications. Early detection is especially important in gallbladder carcinoma, as the prognosis for advanced disease is dismal (5-year survival rate of less than 10\%) [3].

\section{Compliance with ethical standards}

Conflict of interest The author has no conflicts of interest.

Statement of human and animal rights All procedures performed in this study were in accordance with the ethical standards of the institutional and/or national research committee and with the 1964 Helsinki declaration and its later amendments or comparable ethical standards. No animal studies were performed.

Informed consent Informed consent was obtained from all participants included in this study.

\section{References}

1. Stephen AE, Berger DL (2001) Carcinoma in the porcelain gallbladder: a relationship revisited. Surgery 129:699-703

2. Levin B (1999) Gallbladder carcinoma. Ann Oncol 1(10):S129_ S130

3. Misra S, Chaturvedi A, Misra NC et al (2003) Carcinoma of the gallbladder. Lancet Oncol 4:167-176

4. Sheth S, Bedford A, Chopra S (2000) Primary gallbladder cancer: recognition of risk factors and the role of prophylactic cholecystectomy. Am J Gastroenterol 95:1402-1410

5. Khan ZS, Livingston EH, Huerta S (2011) Reassessing the need for prophylactic surgery in patients with porcelain gallbladder: case series and systematic review of the literature. Arch Surg 146:1143-1147 